\section{Retinal sensitivity assessed by microperimetry and corresponding retinal structure and thickness in resolved central serous chorioretinopathy}

HW Chung ${ }^{1}$, CM Yun², JT Kim³ ${ }^{3}$ S-W Kim², $\mathrm{J} \mathrm{Oh}^{2}$ and $\mathrm{K} \mathrm{Huh}{ }^{2}$

\footnotetext{
Abstract

Purpose To investigate the relationship between retinal sensitivity (RS) assessed by microperimetry (MP) and retinal structural changes in patients with resolved central serous chorioretinopathy (CSC).

Methods Spectral domain optical coherence tomography (OCT) examination and MP tests were performed in patients with resolved CSC. Point-to-point correlation was performed between RS and corresponding retinal structural changes using Pearson's correlation analysis. In addition, in a 1-mm zone in the central fovea, a correlation was calculated between the mean RS and the mean central retinal thickness (CRT).

Results Eighty-four eyes were analyzed. The total number of MP test points was 1092 (84 eyes $\times 13$ points). The mean RS and retinal point thickness (RPT) of all test points were $13.53 \pm 3.84 \mathrm{~dB}$ and $208.6 \pm 48.0 \mu \mathrm{m}$, respectively. The RS and RPT were significantly decreased in the test points with loss of the ellipsoid portion of the inner segments (EPIS) $(P<0.0001)$. Within the 1-mm foveal center zone, there was a significant correlation between mean RS and mean CRT ( $r=0.432, P<0.0001)$ and between RS and the corresponding RPT $(r=0.339$, $P<0.0001$ ).

Conclusion RS was dependent on the status of the EPIS in patients with resolved CSC.

The correlation between mean RS and mean CRT was compatible with the point-topoint correlation between RS and the corresponding RPT.
}

Eye (2014) 28, 1223-1230; doi:10.1038/eye.2014.185; published online 1 August 2014

\section{Introduction}

Central serous chorioretinopathy (CSC) is an eye disease characterized by serous retinal detachment caused by leaks from the retinal pigment epithelium (RPE). ${ }^{1-4} \mathrm{CSC}$ is known to be caused primarily by choroidal circulation abnormalities, resulting in choroidal vascular hyperpermeability. ${ }^{1-4}$ The choroidal thickness in eyes with CSC is found to be increased. ${ }^{5}$ The major symptom is decreased visual acuity (VA), although VA may be normal in the early stage of the disease. Other symptoms of CSC are relative scotoma, micropsia, metamorphopsia, and disturbance of color sensation. ${ }^{6}$ CSC has a relatively good long-term prognosis and is self-limited in the majority of patients. ${ }^{7}$ Most retinal detachments in acute CSC resolve spontaneously in 3-6 months or immediately after treatment. ${ }^{8,9}$ However, there may be residual symptoms such as decreased retinal sensitivity, metamorphopsia, decreased VA, and central scotoma. ${ }^{10-12}$ In chronic cases, visual function declines because unresolved retinal detachment can lead to RPE and photoreceptor damage. ${ }^{13,14}$

The usefulness of microperimetry (MP) in evaluating retinal sensitivity (RS) and central retinal field function is well known in resolved CSC. ${ }^{15-24}$ Although most MP evaluations of RS in patients after CSC resolution have used the MP-1 Microperimeter (Nidek Technologies, Padova, Italy), ${ }^{15-24}$ this microperimeter presents
${ }^{1}$ Korea University College of Medicine, Seoul, Korea

${ }^{2}$ Department of Ophthalmology, Korea University College of Medicine, Seoul, Korea

${ }^{3}$ Department of Ophthalmology, Chung-Ang University College of Medicine, Seoul, Korea

Correspondence: S-W Kim, Department of Ophthalmology, Korea University Ansan Hospital, 516 Gojan-dong, Danwon-gu, Ansan-si, Kyung gi-do 425-707, Korea Tel: +82 262463380 Fax: +82 29246820 E-mail: ksw64723@

korea.ac.kr

Received: 22 December 2013

Accepted in revised form: 26 June 2014 Published online: 1 August 2014 
MP values on a confocal scanning laser ophthalmoscope (cSLO) image without producing an optical coherence tomography (OCT) image. Therefore, direct comparisons between RS and retinal changes at a corresponding point are not possible. Some studies have evaluated the relations between RS and OCT findings, but they must compare the two images separately. ${ }^{22-24}$ Spectral OCT/ SLO (OPKO-OTI, Miami, FL, USA) can directly compare $\mathrm{RS}$ and retinal morphology at corresponding points because it also offers an add-in MP test option. The process combines simultaneous high-resolution crosssectional OCT imaging of retinal layers with en face fundus imaging of the retina using the cSLO. Using the built-in instrument software, MP test results are superimposed on an OCT topography scan and analyzed in each patient.

The purpose of the current study was to directly evaluate the relationships between RS and retinal structural changes at corresponding test points in eyes with resolved CSC. We also compared the correlation coefficient between the RS of MP test points and the corresponding retinal point thickness (RPT) with the correlation coefficient between the mean RS and mean central retinal thickness (CRT) at the center of the fovea.

\section{Materials and methods}

Approval was obtained from the Institutional Review Board of the Korea University Medical Center, Seoul, Korea. All research and data collection complied with the Declaration of Helsinki.

\section{Case selection}

A retrospective chart review was performed on patients with resolved CSC at Korea University Ansan Hospital between 1 August 2010 and 31 July 2013. Each patient had a documented episode of CSC diagnosed by fluorescein angiography and indocyanine green angiography. CSC resolution was confirmed with spectral domain OCT (SD-OCT). Eyes were included if at least 4 months had elapsed since the confirmation of CSC resolution by SD-OCT. We excluded patients who were older than 70 years or had other intraocular diseases (that is, age-related macular degeneration, polypoidal choroidal vasculopathy, epiretinal membrane, or diabetic retinopathy). Eyes with a history of ocular surgery, a signal-to-noise ratio $<6 / 10$ on OCT scan, $\leq 50 \%$ of fixation points within $0.5 \mathrm{~mm}$ of the foveal center, or $\leq 75 \%$ of fixation points located within a $2^{\circ}$ circle in the fixation stability test were also excluded. However, eyes with a history of photodynamic therapy (PDT) for CSC or cataract surgery were not excluded.

\section{Microperimetry}

Macular MP was performed using the Spectral OCT/ SLO system. Pupils were dilated, and threshold fundus perimetry was performed on the central $4^{\circ}$ (diameter) of the retina using 13 stimulus points ( 5 cross-shaped points for the central $2^{\circ}$ and 8 ring-shaped points for the central $2-4^{\circ}$ ). Goldmann III size stimuli were projected on a whitish-green background illumination of $1.27 \mathrm{~cd} / \mathrm{m}^{2}$ (4 Asb) with a presentation time of $200 \mathrm{~ms}$ and a $1000-\mathrm{ms}$ interval between stimuli. Stimulus intensity ranged from $0 \mathrm{~dB}\left(127 \mathrm{~cd} / \mathrm{m}^{2}\right)$ to $20 \mathrm{~dB}\left(2.54 \mathrm{~cd} / \mathrm{m}^{2}\right)$. The fixation target used for all subjects was a $1^{\circ}$ single cross. The 4 - to 2-dB staircase threshold strategy was used.

\section{Optical coherence tomography}

SD-OCT was performed using the Spectral OCT/SLO system. All OCT images included line scans (horizontal and vertical B-scans), raster scans, and topographic mapping. Line scans were performed horizontally and vertically, and the scan image was created by averaging up to 20 B-scans (1024 A-scans per B-scan) of $9 \mathrm{~mm}$ in length. The raster scan was performed using $32 \mathrm{~B}$-scans (1024 A-scans per B-scan) of a $9 \times 9 \mathrm{~mm}$ area. The topographic mapping consisted of 256 horizontal A-scans $\times 256$ vertical lines across a $5 \times 5 \mathrm{~mm}$ area. The line scans, raster scans, and topographic mapping were performed on each eye.

\section{Image analysis}

To compare the results of MP values with the OCT scans, OCT topography images and MP scans from each patient were overlaid automatically in the built-in software of the Spectral OCT/SLO system. If the overlaid images did not match well, the authors manually overlaid the two translucent images before final superimposition.

Similarly, to calculate the mean CRT of a 1-mm zone of the foveal center, the authors checked the alignment between the foveal center and the center of the concentric circles and manually aligned the two if needed (Figure 1). Then, the MP test points located within the inner circle were chosen to calculate a mean value within a $1-\mathrm{mm}$ zone around the foveal center. In the OCT topography images, we focused on abnormalities in the outer retina at the MP test point. Reflective line integrities of the ellipsoid portions of the inner segments (EPIS, previously known as the junction between the inner and outer segments of the photoreceptors (IS/OS) $)^{25}$ on the OCT topography scan were assessed qualitatively. We assigned eyes with a defective EPIS signal to the EPIS-damaged group. 


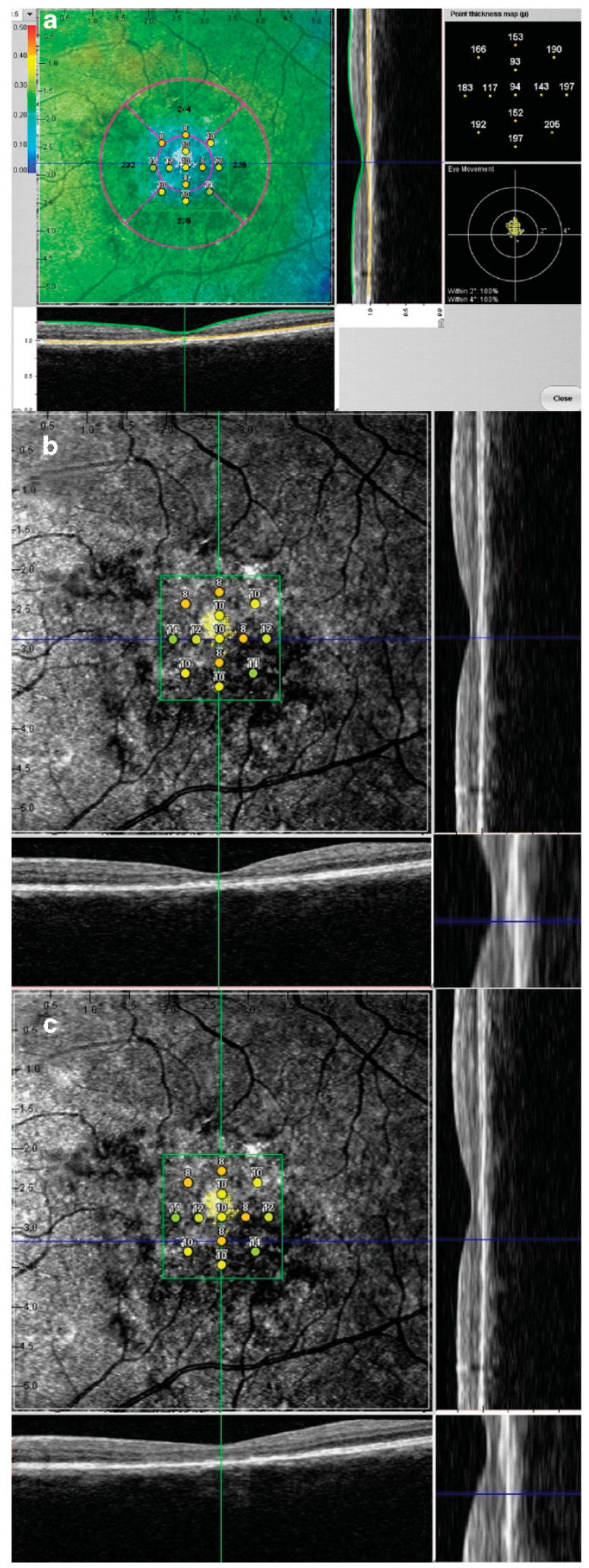

\section{Statistical analysis}

Bivariable analysis using $t$-tests was performed to compare the RS assessed by MP and the retinal thickness according to PDT history and EPIS status. RS and retinal thickness were stratified according to the test location (within vs outside fovea center $1 \mathrm{~mm}$ zone) and then compared between diseased eyes and normal fellow eyes. Pearson's correlation analyses were performed between retinal thickness, RS, and best-corrected visual acuity (BCVA). Statistical analysis was performed using SPSS version 17.0 (SPSS Inc., Chicago, IL, USA). A $P$-value of $<0.05$ was considered statistically significant.

\section{Results}

The total number of eyes of resolved CSC with MP examination was 136. Among these, 52 eyes were excluded. We excluded four eyes because the patients were older than 70 years. Seven eyes were excluded owing to other concurrent intraocular diseases and seven eyes were excluded for a signal-to-noise ratio $<6 / 10$ on OCT scan. We excluded 7 eyes because a different stimulus pattern of MP was used, 6 eyes because they had $\leq 50 \%$ of fixation points within $0.5 \mathrm{~mm}$ of the foveal center, and 12 eyes because they had $\leq 75 \%$ of fixation points located within a $2^{\circ}$ circle in the fixation stability test. MP data for nine eyes could not be extracted in the viewer program because of data loss. Finally, 84 eyes in 77 patients were evaluated, with $41(48.8 \%)$ right and 43 (51.2\%) left eyes, and 27 (31.2\%) eyes with a history of reduced fluence PDT. Among these, 64 eyes could be compared with a normal fellow eye. In the diseased eyes, the total number of test points for MP and RPT were 1092 (84 eyes $\times 13$ points in each eye). The mean age was $47.01 \pm 8.35$ years. The $-\log$ MAR BCVA was $0.10 \pm 0.16$. The mean RS assessed by MP and RPT of all test points

Figure 1 (a) Overlapped map of microperimetry (MP) and optical coherence tomography (OCT) topography of a patient with resolved central serous chorioretinopathy (CSC). The green vertical and blue horizontal lines indicate the planes of the horizontal and vertical retinal cross-sections, which are shown to the right and below the image, respectively. At first, the concentric circle is centered on the foveal center by positioning the two lines on the foveal center at the horizontal and vertical retinal cross-sections. The center test point for MP is slightly below the foveal center. One hundred percent of fixation points were located within $0.5 \mathrm{~mm}$ of the foveal center within a $2^{\circ}$ circle. (b) Scan of the same retina shown in (a). The cross-sections are centered on a point with an MP sensitivity of 10 decibels (dB). The ellipsoid portions of the inner segments (EPIS) are intact in both the horizontal and vertical retinal cross-sections. (c) Scan of the same retina shown in A. The horizontal and vertical cross-sections are centered on a point with an MP sensitivity of $8 \mathrm{~dB}$. The EPIS layer is damaged at this point. 
was $13.53 \pm 3.84 \mathrm{~dB}$ and $208.6 \pm 48.0 \mu \mathrm{m}$, respectively. The mean RS and CRT of a 1-mm zone within the foveal center was $12.95 \pm 3.78 \mathrm{~dB}$ and $177.9 \pm 33.3 \mu \mathrm{m}$, respectively. The number of damaged EPIS was 126 (11.54\%; Table 1). There was no significant difference in - logMAR BCVA and mean RS within fovea center $1 \mathrm{~mm}$ zone between eyes with and without a history of PDT. However, the CRT of a 1-mm zone within the foveal center was significantly thinner in eyes with a history of PDT (183.4 \pm 33.1 vs $166.2 \pm 31.3 \mu \mathrm{m}, P=0.026$; Table 2$)$. Compared with the intact EPIS group, the RS of the damaged EPIS group showed an average of $5.97 \mathrm{~dB}$ lower sensitivity $(P<0.0001)$ and $38.8 \mu \mathrm{m}$ decreased RPT $(P<0.0001$; Table 3). The RS and RPT of the diseased eye were significantly lower than those of the normal fellow eye both within and outside the fovea center $1 \mathrm{~mm}$ zone (Table 4).

The - logMAR BCVA was significantly correlated with both mean RS and CRT within fovea center $1 \mathrm{~mm}$ zone $(r=-0.542, P<0.0001$, and $r=-0.412, P<0.0001$, respectively). The correlation coefficient between RS and $\mathrm{RPT}$ at all test points was $0.348(P<0.0001)$. After excluding the effect of EPIS damage and test location, the partial correlation coefficient between RS and RPT at all test points was decreased $(r=0.229, P<0.0001)$. Within the foveal center, the correlation coefficients between the mean RS and the mean CRT, and between individual RS and RPT were $0.432(P<0.0001)$ and $0.348(P<0.0001)$, respectively. The correlation coefficient between the RS and RPT stratified by the status of EPIS (present and absent) was $0.259(P<0.0001)$ in the intact EPIS group and $0.305(P=0.001)$ in the damaged EPIS group. The correlation coefficient between the RS and RPT stratified by test location was $0.339(P<0.0001)$ within the foveal center and $0.329(P<0.0001)$ outside it (Table 5). A positive association was observed with a lowess curve in the scatter plots of the relationship between RS and RPT (Figure 2).

\section{Discussion}

In the present study, the RS and RPT were significantly lower in the damaged EPIS group. RPT also had a significant correlation with RS. Interestingly, in the 1-mm central foveal zone, the correlation between mean RS and mean CRT was slightly higher than the point-to-point correlation (0.432 vs 0.339). This result implies that, when evaluating the correlation between RS and retinal thickness, there is no need to manually measure the point-to-point relation; it may suffice to simply use the automatically calculated mean RS and CRT in the software. In the previous study with spectral OCT/SLO, the correlation between CRT at the foveal center and RS was 0.316 at the center $2^{\circ}$ and 0.493 at the center $4^{\circ} .^{26}$
Table 1 Baseline characteristics of patients $(n=84)$ and examined retinal points $(n=1092)$

\begin{tabular}{|c|c|}
\hline$\overline{\text { Age (years) }}$ & $47.01 \pm 8.35$ \\
\hline Male/female & $71(84.5 \%) / 13(15.5 \%)$ \\
\hline Right eye/left eye & $41(48.8) / 43(51.2 \%)$ \\
\hline History of PDT (no/yes) & $57(67.9 \%) / 27(32.1 \%)$ \\
\hline BCVA $(-\log M A R)$ & $0.104 \pm 0.159$ \\
\hline Central retinal thickness $(\mu \mathrm{m})^{a}$ & $177.9 \pm 33.3$ \\
\hline Retinal sensitivity $(\mathrm{dB})^{\mathrm{b}}$ & $13.53 \pm 3.84$ \\
\hline Retinal point thickness $(\mu \mathrm{m})^{\mathrm{b}}$ & $208.6 \pm 48.0$ \\
\hline EPIS damage ${ }^{b}$ & $126(11.54 \%)$ \\
\hline
\end{tabular}

Abbreviations: BCVA, best-corrected visual acuity; EPIS, the ellipsoid portion of the inner segments; logMAR, logarithm of the minimum angle of resolution; PDT, photodynamic therapy.

${ }^{a}$ Measured at a 1-mm zone within the foveal center on the topography map of optical coherence tomography.

${ }^{b}$ Measured at all microperimetry points $(84$ eyes $\times 13$ test points $=1092$ points).

Table 2 Comparison of BCVA, RS, and retinal thickness according to the history of half-fluence PDT (mean \pm SD)

\begin{tabular}{lrrr}
\hline & \multicolumn{1}{c}{$\begin{array}{c}P D T(-) \\
(\mathrm{n}=57)\end{array}$} & \multicolumn{1}{c}{$\begin{array}{c}\text { PDT }(+) \\
(\mathrm{n}=27)\end{array}$} & P-value $^{\mathrm{a}}$ \\
\hline BCVA ( - logMAR) & $0.10 \pm 0.15$ & $0.11 \pm 0.17$ & 0.789 \\
RS $(\mathrm{dB})$ & $13.18 \pm 2.95$ & $12.49 \pm 3.25$ & 0.332 \\
CRT $(\mu \mathrm{m})$ & $183.4 \pm 33.1$ & $166.2 \pm 31.3$ & 0.026 \\
\hline
\end{tabular}

Abbreviations: BCVA, best-corrected visual acuity; CRT, central retinal thickness at fovea center $1 \mathrm{~mm}$ zone; logMAR, logarithm of the minimum angle of resolution; PDT, photodynamic therapy; RS, retinal sensitivity at fovea center $1 \mathrm{~mm}$ zone.

${ }^{\text {a }}$ Independent $t$-test.

Table 3 Retinal sensitivity and retinal point thickness based on the status of the EPIS (mean \pm SD)

\begin{tabular}{lrrrr}
\hline & $\begin{array}{c}\text { Retinal point } \\
\text { sensitivity } \\
(d B)\end{array}$ & P-value & $\begin{array}{c}\text { Retinal point } \\
\text { thickness } \\
(\mu \mathrm{m})\end{array}$ & P-value \\
\hline All $(n=1092)$ & $13.53 \pm 3.84$ & & $208.6 \pm 48.0$ & \\
& & & & \\
EPIS damage & & & & \\
No $(n=966)$ & $14.22 \pm 3.01$ & $<0.0001$ & $213.1 \pm 46.3$ & $<0.0001$ \\
Yes $(n=126)$ & $8.25 \pm 5.23$ & & $174.3 \pm 46.9$ & \\
\hline
\end{tabular}

Abbreviation: EPIS, the ellipsoid portion of the inner segments. ${ }^{a}$ Independent $t$-test.

However, the relationship between the fixation center of MP and the anatomical foveal center was not considered, so a mean RS at the center $2^{\circ}$ did not always reflect the RS at the 1-mm zone of the foveal center in that study. Oh et $a^{27}$ demonstrated that fixation during SD-OCT scanning was frequently eccentric in other diseases and might be dependent on photoreceptor integrity. In the present study, eyes with $<50 \%$ of fixation points within 
Table 4 Comparison of retinal sensitivity and retinal point thickness between diseased eyes and normal fellow eyes (mean \pm SD)

\begin{tabular}{lccc}
\hline$n=64$ & Diseased eye & Normal fellow eye & P-value \\
\hline RS within fovea center 1 mm zone $(\mathrm{dB})$ & $12.79 \pm 3.16$ & $14.87 \pm 2.43$ & $<0.0001$ \\
RS outside fovea center 1 mm zone $(\mathrm{dB})$ & $13.92 \pm 2.77$ & $16.00 \pm 2.18$ & $<0.0001$ \\
RS of central $4^{\circ}$ area $(\mathrm{dB})$ & $13.47 \pm 2.66$ & $15.51 \pm 2.10$ & $<0.0001$ \\
RPT within fovea center $1 \mathrm{~mm}$ zone $(\mu \mathrm{m})$ & $173.7 \pm 36.6$ & $205.2 \pm 29.5$ & $<0.0001$ \\
RPT outside fovea center $1 \mathrm{~mm}$ zone $(\mu \mathrm{m})$ & $229.3 \pm 36.2$ & $260.0 \pm 2.2$ & $<0.0001$ \\
RPT of central $4^{\circ}$ area $(\mu \mathrm{m})$ & $205.4 \pm 35.9$ & $235.9 \pm 26.6$ & $<0.0001$ \\
Central retinal thickness $(\mu \mathrm{m})^{\mathrm{b}}$ & $173.7 \pm 36.3$ & $206.3 \pm 28.5$ & $<0.0001$ \\
\hline
\end{tabular}

Abbreviations: RPT, retinal point thickness; RS, retinal sensitivity. Retinal sensitivity was assessed by microperimetry.

a Paired $t$-test.

${ }^{b}$ Measured at a 1-mm zone within the foveal center on the topography map of optical coherence tomography.

Table 5 Pearson's correlation analyses among BCVA, RS assessed by microperimetry, and retinal thickness

\begin{tabular}{lcc}
\hline & $\mathrm{r}$ & $\mathrm{P}$ \\
\hline BCVA vs mean RS at fovea center & mm zone & \\
All eyes $(n=84)$ & -0.542 & $<0.0001$ \\
& & \\
BCVA vs mean CRT & -0.412 & $<0.0001$ \\
All eyes $(n=84)$ & & \\
& & \\
Mean CRT vs mean RS at fovea center 1 mm zone & \\
All eyes $(n=84)$ & 0.432 & \\
& & $<0.0001$ \\
RS vs retinal point thickness & & \\
All test points $(n=1092)$ & 0.348 & $<0.0001$ \\
& $0.229^{\mathrm{a}}$ & $<0.0001$ \\
EPIS damage $(-)(n=966)$ & 0.259 & 0.001 \\
EPIS damage $(+)(n=126)$ & 0.305 & $<0.0001$ \\
Within 1 mm zone $(n=478)$ & 0.339 & $<0.0001$ \\
Outside 1 mm zone $(n=614)$ & 0.329 & \\
\hline
\end{tabular}

Abbreviations: BCVA, best-corrected visual acuity ( - logarithm of the minimum angle of resolution); CRT, central retinal thickness at fovea center $1 \mathrm{~mm}$ zone; EPIS, the ellipsoid portion of the inner segments; RS, retinal sensitivity.

a Partial correlation after excluding the effect of EPIS damage and test location (within $v s$ outside the central fovea).

$0.5 \mathrm{~mm}$ of the foveal center were excluded, and MP test points located in the foveal center were correlated with CRT. Vasconcelos et $a l^{22}$ reported that the central $2^{\circ}$ RS (with MP-1) was highly correlated with central macular thickness (Pearson $=0.87, P<0.001$; Spearman's rho $=0.90, P<0.001)$ in resolved CSC. On the other hand, Reibaldi et al ${ }^{18}$ could not find a significant correlation between RS (with MP-1) and central foveal thickness after PDT in chronic CSC. The authors attributed their findings to the presence of subretinal fluid (persistent or recurrent) in some patients.

There are also many studies regarding RS and the status of the retinal microstructure on OCT after CSC resolution. ${ }^{22-24} \mathrm{RS}$ was compared according to the status of the external limiting membrane (ELM), the IS/OS line, the cone outer segment tips (COST) line, and the RPE line. Vasconcelos et $a l^{22}$ reported that, in the central $12^{\circ}$ and $2^{\circ}$, RS was statistically correlated with morphological changes in the ELM and IS/OS line but not with RPE changes after PDT for chronic CSC.

However, Ojima et $a l^{23}$ suggested that both the irregular RPE line and defects of the IS/OS line were associated with decreased RS after CSC resolution. In a study by Fujita et al, ${ }^{24}$ the RS was significantly higher in the IS/OS $(+)$ COST $(+)$ group than in the IS/OS $(+)$ COST $(-)$ group after PDT for chronic CSC. In our previous reports, ${ }^{26,28,29}$ the correlations among different tests were evaluated in resolved CSC. RS at the fovea $\left(2^{\circ}\right)$ was significantly associated with the IS/OS defect in OCT and $\mathrm{N} 1$ latency at R1 by multifocal electroretinography. ${ }^{26}$ Visual function (vision and RS) did not correlate well with near-infrared autofluorescent (AF) gray value parameters, but was moderately correlated with shortwavelength AF gray value parameters. ${ }^{28}$ There was a significant association between the OCT and shortwavelength AF findings. An abnormal short-wavelength AF signal was significantly associated with loss of the EPIS on OCT. However, near-infrared AF could not predict the status of EPIS without the short-wavelength AF image. ${ }^{29}$

In the present study, eyes with reduced fluence PDT showed thinner CRT than eyes with a history of spontaneous resolution of CSC, even though there was no significant difference in BCVA or RS between the two groups. This difference in CRT might be due to baseline differences before treatment or the effect of PDT. ${ }^{30}$ The RS and RPT of the diseased eye were significantly lower than those of the normal fellow eye both within and outside the fovea center $1 \mathrm{~mm}$ zone. In the normal fundus, the retinal thickness is shorter in the fovea than in the perifovea. The RS assessed by MP was known to be the same within the central $6^{\circ}$ of the macula in normal subjects. ${ }^{31}$ Therefore, the correlation analysis between RS and RPT needs to be stratified according to the test location. The correlation coefficient between RS and RPT decreased after controlling for EPIS status and test location. 

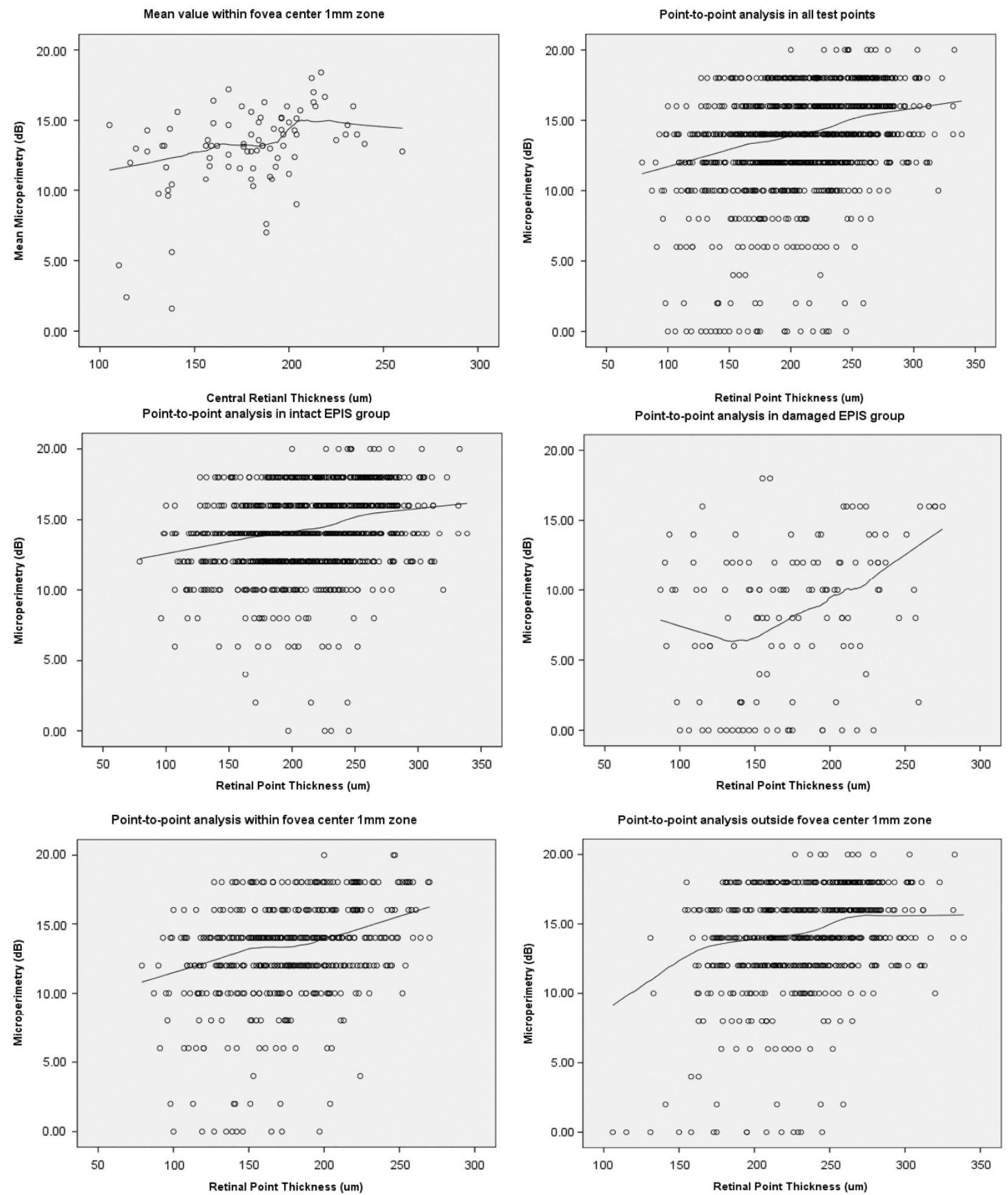

Figure 2 Scatter plots of retinal sensitivity $(\mathrm{dB})$ assessed by microperimetry $v$ s retinal point thickness $(\mu \mathrm{m})$ stratified by the status of ellipsoid portion of the inner segments and MP test location (within vs outside fovea center $1 \mathrm{~mm}$ zone). A lowess curve was fit in all graphs (black line).

This might be because RS and retinal thickness were significantly dependent on the status of the EPIS, test point, and anatomical location.
We observed a correlation between RS and RPT, and damage to the EPIS was correlated with worse RS in the scatter plots; however, there was also ample scatter 
(spread) in the data, which could imply individual variation. Therefore, it is difficult to draw meaningful conclusions from variations in these correlation coefficients and to propose a hypothesis to explain these variations. However, it is worthwhile to consider that, because there was no macular swelling and all RPTs were within or below the normal range in resolved CSC, the correlation might be very weak in both the intact and the damaged EPIS groups.

There are several limitations to the present study. First, the number of participants was relatively small, and the study design was retrospective. Second, because eyes with good and stable foveal fixation were included, selection bias could have affected the results, although more reliable test results were obtained. All cases had a BCVA $>0.5$. Third, because the OCT topography mapping consisted of 256 horizontal A-scans $\times 256$ vertical lines across a $5 \times 5 \mathrm{~mm}$ area, the EPIS in some cases was difficult to classify as damaged or not damaged. Finally, the number of test points in each eye was only 13 , so even though the points were concentrated within the central $4^{\circ}$ of the retina, the mean RS value may not depend on general retinal function.

In conclusion, RS assessed by MP was dependent on the status of the EPIS in patients with resolved CSC. The correlation between mean RS and mean CRT was compatible with the point-to-point correlation between RS and the corresponding RPT.

\section{Summary}

\section{What was known before}

- After CSC resolution, RS at the fovea had a significant association with the IS/OS defect in OCT.

\section{What this study adds}

- In resolved CSC, the correlation between mean RS and mean CRT was compatible with the point-to-point correlation between RS and the corresponding RPT.

\section{Conflict of interest}

The authors declare no conflict of interest.

\section{Acknowledgements}

JO received support from the Korean Health Technology R\&D Project, Ministry for Health, Welfare \& Family Affairs, Republic of Korea (A102024).

\section{Disclaimer}

The funding organization had no role in the design or conduct of this research.

\section{References}

1 Prunte C. Indocyanine green angiographic findings in central serous chorioretinopathy. Int Ophthalmol 1995; 19(2): 77-82.

2 Prunte C, Flammer J. Choroidalcapillary and venous congestion in central serous chorioretinopathy. Am J Ophthalmol 1996; 121(1): 26-34.

3 Kitaya N, Nagaoka T, Hikichi T, Sugawara R, Fukui K, Ishiko $\mathrm{S}$ et al. Features of abnormal choroidal circulation in central serous chorioretinopathy. Br J Ophthalmol 2003; 87(6): 709-712.

4 Guyer DR, Yannuzzi LA, Slakter JS, Sorenson JA, Ho A, Orlock D. Digital indocyanine green video angiography of central serous chorioretinopathy. Arch Ophthalmol 1994; 112(8): 1057-1062.

5 Kim SW, Oh J, Kwon SS, Yoo J, Huh K. Comparison of choroidal thickness among patients with healthy eyes, early age-related maculopathy, neovascular age-related macular degeneration, central serous chorioretinopathy, and polypoidal choroidal vasculopathy. Retina 2011; 31(9): 1904-1911.

6 Klais CM, Ober MD, Ciardella AP, Yannuzzi LA. Central serous chorioretinopathy. In: Ryan SJ (ed). Retina, 4th edn. Elsevier Mosby: Philadelphia, PA, USA, 2006, p 1140.

7 Levine R, Brucker AJ, Robinson F. Long-term follow-up of idiopathic central serous chorioretinopathy by fluorescein angiography. Ophthalmology 1989; 96(6): 854-859.

8 Ahn SE, Oh J, Oh JH, Oh IK, Kim SW, Huh K. Three-dimensional configuration of subretinal fluid in central serous chorioretinopathy. Invest Ophthalmol Vis Sci 2013; 54(9): 5944-5952.

9 Cardillo Piccolino F, Eandi CM, Ventre L, Rigault de la Longrais RC, Grignolo FM. Photodynamic therapy for chronic central serous chorioretinopathy. Retina 2003; 23(6): 752-763.

10 Wang M, Birgit S, Mortenla C, Michael L. Clinical characteristics of subretinal deposits in central serous chorioretinopathy. Acta Ophthalmol Scand 2005; 83(6): 691-696.

11 Gackle HC, Lang GE, Freißler KA, Lang GK. Clinical, fluorescein angiographic and demographic aspects in central serous chorioretinopathy. Ophthalmologe 1998; 95(8): 529-533.

12 Baran NV, Gürlü VP, Esgin H. Long-term macular function in eyes with central serous chorioretinopathy. Clin Experiment Ophthalmol 2005; 33(4): 369-372.

13 Gilbert CM, Owens SL, Smith PD, Fine SL. Long-term follow-up of central serous chorioretinopathy. $\mathrm{Br} J$ Ophthalmol 1984; 68(11): 815-820.

14 Wang MS, Sander B, Larsen M. Retinal atrophy in idiopathic central serous chorioretinopathy. Am J Ophthalmol 2002; 133(6): 787-793.

15 Ozdemir H, Karacorlu SA, Senturk F, Karacorlu M, Uysal O. Assessment of macular function by microperimetry in unilateral resolved central serous chorioretinopathy. Eye (Lond) 2008; 22(2): 204-208.

16 Fujita K, Shinoda K, Matsumoto CS, Imamura Y, Tanaka E, Mizutani $\mathrm{Y}$ et al. Microperimetric evaluation of chronic central serous chorioretinopathy after half-dose photodynamic therapy. Clin Ophthalmol 2012; 6: 1681-1687.

17 Ehrlich R, Mawer NP, Mody CH, Brand CS, Squirrell D. Visual function following photodynamic therapy for central serous chorioretinopathy: a comparison of automated 
macular microperimetry versus best-corrected visual acuity. Clin Experiment Ophthalmol 2012; 40(1): e32-e39.

18 Reibaldi M, Boscia F, Avitabile T, Uva MG, Russo A, Zagari $\mathrm{M}$ et al. Functional retinal changes measured by microperimetry in standard-fluence vs low-fluence photodynamic therapy in chronic central serous chorioretinopathy. Am J Ophthalmol 2011; 151(6): 953-960.

19 Senturk F, Karacorlu M, Ozdemir H, Karacorlu SA, Uysal O. Microperimetric changes after photodynamic therapy for central serous chorioretinopathy. Am J Ophthalmol 2011; 151(2): 303-309.

20 Fujita K, Yuzawa M, Mori R. Retinal sensitivity after photodynamic therapy with half-dose verteporfin for chronic central serous chorioretinopathy: short-term results. Retina 2011; 31(4): 772-778.

21 Pryds A, Larsen M. Foveal function and thickness after verteporfin photodynamic therapy in central serous chorioretinopathy with hyperautofluorescent subretinal deposits. Retina 2013; 33(1): 128-135.

22 Vasconcelos H, Marques I, Santos AR, Melo P, Pires I, Figueira $\mathrm{J}$ et al. Long-term chorioretinal changes after photodynamic therapy for chronic central serous chorioretinopathy. Graefes Arch Clin Exp Ophthalmol 2013; 251(7): 1697-1705.

23 Ojima Y, Tsujikawa A, Hangai M, Nakanishi H, Inoue R, Sakamoto A et al. Retinal sensitivity measured with the micro perimeter 1 after resolution of central serous chorioretinopathy. Am J Ophthalmol 2008; 146(1): 77-84.

24 Fujita K, Shinoda K, Imamura Y, Matsumoto CS, Mizutani Y, Mizota A et al. Correlation of integrity of cone outer segment tips line with retinal sensitivity after half-dose photodynamic therapy for chronic central serous chorioretinopathy. Am J Ophthalmol 2012; 154(3): 579-585.

25 Spaide RF, Curcio CA. Anatomical correlates to the bands seen in the outer retina by optical coherencetomography: literature review and model. Retina 2011; 31(8): 1609-1619.

26 Kim SW, Oh J, Huh K. Correlations among various functional and morphological tests in resolved central serous chorioretinopathy. Br J Ophthalmol 2012; 96(3): 350-355.

27 Oh IK, Oh J, Kim SW, Huh K. Fixation and photoreceptor integrity in optical coherence tomography. Optom Vis Sci 2012; 89(7): E1000-E1008.

28 Oh J, Kim SW, Kwon SS, Oh IK, Huh K. Correlation of fundus autofluorescence gray values with vision and microperimetry in resolved central serous chorioretinopathy. Invest Ophthalmol Vis Sci 2012; 53(1): 179-184.

29 Kim SK, Kim SW, Oh J, Huh K. Near-infrared and shortwavelength autofluorescence in resolved central serous chorioretinopathy: association with outer retinal layer abnormalities. Am J Ophthalmol 2013; 156(1): 157-164.

30 Copete S, Ruiz-Moreno JM, Cava C, Montero JA. Retinal thickness changes following photodynamic therapy in chronic central serous chorioretinopathy. Graefes Arch Clin Exp Ophthalmol 2012; 250(6): 803-808.

31 Shah VA, Chalam KV. Values for macular perimetry using the MP-1 microperimeter in normal subjects. Ophthalmic Res 2009; 41(1): 9-13. 